\title{
Meia-vida do diuron em solos com diferentes atributos físicos e químicos
}

\author{
Half - life of diuron in soils with different physical and chemical attributes
}

\author{
Paulo Roberto Ribeiro Rocha ${ }^{\mathrm{I}}$ Autieres Teixeira Faria ${ }^{\mathrm{II}}$ Gustavo Soares da Silva ${ }^{\mathrm{II}}$ \\ Maria Eliana Lopes Ribeiro de Queiroz ${ }^{\text {II }}$ Franklyn Clawdy Nunes GuimarãesII \\ Siumar Pedro Tironi ${ }^{I I I}$ Leandro Galon ${ }^{\text {IV }}$ Antonio Alberto da Silva ${ }^{\text {II }}$
}

RESUMO

A distribuição e a dissipação dos herbicidas nos solos são processos dinâmicos e únicos para cada relação soloherbicida. Neste trabalho, a meia-vida do diuron foi determinada em cinco solos (Latossolo Vermelho Amarelo, Latossolo Vermelho e Latossolo Amarelo), com diferentes valores de $\mathrm{pH}$. As concentrações do diuron foram determinadas em amostras dos referidos solos coletadas em oito épocas $(1,8,15,22,36$, 66, 96 e 156 dias após a aplicação do herbicida na dose $3,0 \mathrm{~kg}$ $\left.\mathrm{ha}^{-1}\right)$. Em uma parte dessas amostras, foi feita a quantificação do diuron por cromatografia líquida de alta eficiência e, na outra parte, os testes biológicos, visando à confirmação dos resultados das análises cromatográficas. Constatou-se que a persistência do diuron é dependente dos atributos físicos e químicos dos solos. Todavia, a correção do $\mathrm{pH}$ do solo favoreceu apenas a degradação do herbicida em solos arenosos com baixo teor de matéria orgânica. A meia-vida do diuron nos solos estudados variou de 40 a 91 após a sua aplicação, sendo maior no solo com maior teor de matéria orgânica. Esses resultados foram confirmados pelos testes biológicos que se mostraram eficientes e complementares às análises cromatográficas.

Palavras-chave: persistência, herbicidas não-iônicos, bioensaio, cromatografia, latossolos.

\section{ABSTRACT}

The distribution and dissipation of herbicides on soils are dynamic and unique processes for each soil-herbicide relation. In this research, the half-life of diuron was determined in five soils (Red-Yellow Latosol, Red Latosol, and Yellow Latosol), with different $\mathrm{pH}$ values. Diuron concentrations were determined in samples of each soil collected at eight different times $(1,8,15,22,36,66,96$ and 156 days after herbicide application at $\left.3.0 \mathrm{~kg} \mathrm{ha}^{-1}\right)$. Part of each soil sample was used to quantify diuron by high performance liquid chromatography. The other part of soil sample was used to do biological tests in order to confirm the results of chromatographic analysis. It was found that the persistence of diuron is dependent on the physical and chemical soil attributes. However, the correction of soil pH favored only herbicide degradation in sandy soils with low organic matter content. The half-life of diuron in soils ranged from 40 to 91 after its application and it was higher in soil with higher organic matter content. The biological tests confirmed these results and they were efficient and complementary to the chromatographic analysis.

Key words: persistence, non-ionic herbicides, bioassay, chromatography, latosols.

\section{INTRODUÇÃO}

Os agrotóxicos são ferramentas utilizadas nos sistemas de produção agrícola essenciais para a redução das perdas causadas por pragas, doenças e plantas daninhas. Dentre esses compostos, destacamse os herbicidas que representam $40 \%$ do mercado brasileiro (SINDAG, 2011). Entretanto, a utilização desses agrotóxicos sem o conhecimento prévio de suas interações com o solo e clima pode representar redução na eficiência técnica bem como aumentar o risco de contaminação ambiental.

Dentre os herbicidas de grande uso no Brasil, destaca-se o diuron [N-(3,4dichlorophenyl)-N, N-dimethylurea]. Este apresenta amplo espectro de ação, é registrado para o controle de mono e dicotiledôneas e é aplicado em pré e pós-

IUniversidade Federal de Roraima (UFRR), 69300-000, Boa Vista, RR, Brasil. E-mail: paulo.ribeiro.rocha@hotmail. Autor para correspondência.

IDDepartamento de Fitotecnia, Universidade Federal de Viçosa (UFV), Viçosa, MG, Brasil.

IIICentro de Ciências Agrárias (CECA), Universidade Federal de Alagoas (UFAL), Rio Largo, AL, Brasil.

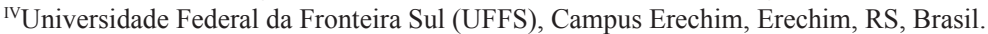


emergência inicial das plantas daninhas. O diuron pertence ao grupo químico das ureias substituídas, apresenta baixa solubilidade em água $42 \mathrm{mg} \mathrm{L}^{-1}$ a $25^{\circ} \mathrm{C}$, pKa igual a zero e Kow de 589 e Koc médio de $480 \mathrm{~mL} \mathrm{~g}^{-1}$ (RODRIGUES \& ALMEIDA, 2011). A maioria dos herbicidas derivados desse grupo químico apresenta elevada persistência no ambiente (HAGER \& NORDBY, 2004). A presença do diuron no solo até 267 dias após a aplicação, em condições de campo, foi verificada por STORK et al. (2008).

A grande importância de se conhecer as interações dos herbicidas com o solo se justifica porque independente dos produtos serem aplicados diretamente no solo ou na parte aérea das plantas, esses acabam por alcançar direta ou indiretamente o solo (MANCUSO et al., 2011). Nos solos, a dissipação dos herbicidas é influenciada pelas características das moléculas, dos solos e pelas interações soloherbicida e clima. $\mathrm{O}$ desconhecimento da persistência dos herbicidas no solo poderá ter como consequência a injúria para as culturas em sucessão, a perda da biodiversidade, além do risco de contaminação de águas superficiais e subterrâneas (COBBUCI \& MACHADO, 1999; FERRI \& VIDAL, 2003).

Os processos de dissipação dos herbicidas no solo podem ser químicos, físicos ou biológicos, resultando na mineralização total do pesticida ou na sua conversão em metabólitos (NAKAGAWA \& ANDRÉA, 2000). A dissipação desses compostos depende da estrutura da molécula e das condições edafoclimáticas. No caso específico do diuron, sua principal via de degradação no solo é a microbiológica (CARACCIOLO, et al., 2005).

Os processos de distribuição e degradação de herbicidas no solo são dinâmicos e únicos para cada relação solo-herbicida. Considerando que o diuron é muito utilizado no Brasil e há carência de informações sobre o seu comportamento em solos tropicais, realizou-se este trabalho com objetivo de determinar a meia-vida desse herbicida em Latossolos, coletados em diferentes regiões brasileiras, com e sem correção de $\mathrm{pH}$.

\section{MATERIAL E MÉTODOS}

O experimento foi conduzido em casa de vegetação, no Departamento de Fitotecnia da Universidade Federal de Viçosa. Foram utilizados três Latossolos de diferentes localidades: Latossolo Amarelo (LA) do município de Sooretama - ES; Latossolo Vermelho-Amarelo (LVA) do município de Viçosa - MG; e Latossolo Vermelho (LV) município de Três Marias - MG. As amostras dos solos foram coletadas na profundidade de 0 a $20 \mathrm{~cm}$.

Foram utilizados solos com dois valores de $\mathrm{pH}$, sem a correção da acidez ( $\mathrm{pH}$ natural) e com a correção (pH próximo a 6,0), com exceção do LA, cujo $\mathrm{pH}$ encontrava-se próximo de $6,0 \mathrm{em}$ condições naturais. $\mathrm{O} \mathrm{pH}$ foi corrigido, com base em curvas de neutralização com $\mathrm{CaCO}_{3}$, para cada Latossolo. Os atributos químicos e físicos dos solos estão apresentadas na tabela 1 .

Utilizou-se o delineamento inteiramente casualizado no esquema fatorial $5 \times 8$, sendo cinco solos (LVA pH 5,0; LVA pH 5,9; LV pH 5,0; LV pH 6,2 e LA pH 6,3) e oito épocas de coleta $[1,8,15$, $22,36,66,96$ e 156 dias após o tratamento (DAT)] com três repetições, além das testemunhas para cada solo onde não houve aplicação do herbicida. As unidades experimentais foram constituídas por vasos com capacidade de $0,33 \mathrm{dm}^{3}$, preenchidos com os respectivos solos. Posteriormente, foi feita a aplicação do diuron na parte superior dos vasos (superfície do solo) em concentração equivalente à dosagem de $6,0 \mathrm{~L} \mathrm{ha}^{-1}$ do produto comercial (Diuron Nortox $500 \mathrm{SC}$ ) contendo $500 \mathrm{~g} \mathrm{~L}^{-1}$ do ingrediente ativo. Utilizou-se um pulverizador pressurizado a $\mathrm{CO}_{2}$, equipado com dois bicos TT 110.02, espaçados de $0,5 \mathrm{~m}$, mantidos à pressão de $25 \mathrm{lb} \mathrm{pol}^{-2}$ e volume de calda de $150 \mathrm{~L} \mathrm{ha}^{-1}$.

Tabela 1 - Resultados das análises químicas e físicas das amostras dos Latossolos antes e depois da correção do pH.

\begin{tabular}{|c|c|c|c|c|c|c|c|c|c|c|c|c|c|c|}
\hline \multirow{2}{*}{ Solos } & $\mathrm{pH}$ & $\mathrm{P}$ & $\mathrm{K}$ & $\mathrm{Ca}^{2+}$ & $\mathrm{Mg}^{2+}$ & $\mathrm{Al}^{3+}$ & $\mathrm{H}+\mathrm{Al}$ & $\mathrm{CTC}(\mathrm{t})$ & V & $\mathrm{m}$ & MO & Areia & Silte & Argila \\
\hline & $\mathrm{H}_{2} \mathrm{O}$ & \multicolumn{2}{|c|}{----mg dm ${ }^{-3}---$} & \multicolumn{5}{|c|}{ 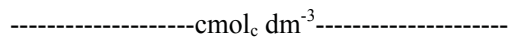 } & \multicolumn{2}{|c|}{-----0\%---- } & \multicolumn{4}{|c|}{-----------------dag kg ${ }^{-1}$--------------- } \\
\hline LVA & 5,0 & 3,5 & 50 & 0,8 & 0,3 & 0,8 & 8,91 & 2,18 & 13 & 37 & 3,7 & 27 & 4 & 69 \\
\hline LVA & 5,9 & 3,5 & 50 & 3,5 & 0,3 & 0 & 8,25 & 3,83 & 32 & 0 & 3,7 & 27 & 4 & 69 \\
\hline LV & 5,0 & 0,8 & 14 & 0,2 & 0,0 & 0,4 & 3,30 & 0,64 & 7 & 63 & 0,8 & 72 & 1 & 27 \\
\hline LV & 6,2 & 0,8 & 14 & 1,1 & 0,0 & 0,0 & 1,48 & 1,16 & 44 & 0 & 0,8 & 72 & 1 & 27 \\
\hline LA & 6,3 & 9,6 & 110 & 2,9 & 1,0 & 0,0 & 1,32 & 4,18 & 76 & 0 & 2,2 & 79 & 1 & 20 \\
\hline
\end{tabular}

LVA - Latossolo Vermelho-Amarelo; LV - Latossolo Vermelho e LA - Latossolo Amarelo. CTC (t) = capacidade de troca catiônica efetiva; $\mathrm{V}=$ saturação por bases; $\mathrm{m}=$ Saturação por $\mathrm{Al}^{+3} ; \mathrm{MO}=$ matéria orgânica. 
Nas épocas de coleta, o solo contido em cada vaso foi homogeneizado e uma amostra de $0,05 \mathrm{dm}^{-3}$ foi retirada para extração e quantificação do herbicida por cromatografia líquida de alta eficiência (CLAE). Posteriormente, semearam-se três sementes por vaso da espécie bioindicadora pepino (Cucumis sativus L.). Aos 21 dias após a emergência (DAE), o índice de intoxicação das plantas foi avaliado atribuindo notas de 0 (ausência de intoxicação) a 100 (morte da planta), de acordo com escala da EWRC (1964) modificada. Em seguida, as plantas foram seccionadas rente ao solo, acondicionadas em sacos de papel e secas em estufa de circulação forçada de ar $\left(70 \pm 2^{\circ} \mathrm{C}\right)$ até atingir massa constante, sendo determinada a matéria seca das plantas.

A extração do diuron do solo foi realizada de acordo com a metodologia descrita por KASOZI et al. (2010). Foram colocados $5 \mathrm{~g}$ de solo seco, em tubos plásticos de fundo cônico e tampa rosqueável, seguido da adição de $20 \mathrm{~mL}$ de metanol aos tubos. Estes foram submetidos à agitação vertical por 3 horas. Em seguida, foram centrifugados por $7 \mathrm{~min}$. a $2.260 \mathrm{~g}$, para a decantação das partículas e limpeza do extrato. O sobrenadante foi retirado e ao resíduo foram adicionados mais $10 \mathrm{~mL}$ de metanol. Os tubos foram novamente agitados (2h) e centrifugados ( $7 \mathrm{~min}$.) e os extratos das duas etapas foram combinados $(20+10 \mathrm{~mL}$ de metanol). Retirou-se $1,5 \mathrm{~mL}$ da mistura dos extratos, filtrou-se em membrana de $0,45 \mu \mathrm{m}$ de poro, diretamente em vidros "vials", os quais foram submetidos à análise por CLAE.

A determinação do diuron foi realizada utilizando-se um sistema de cromatografia líquida de alta eficiência, modelo Shimadzu LC 20AT, detector UV-Vis (Shimadzu SPD 20A), coluna $\mathrm{C}_{18}$ de aço inox (Shimadzu VP-ODS Shim-pack 150mmx4,6mm d.i.). A solução estoque do herbicida foi preparada a partir do padrão com $99,0 \%$ de pureza, na concentração de $1.000 \mathrm{mg} \mathrm{L}^{-1}$ em metanol e as soluções de trabalho preparadas a partir desta.

As condições cromatográficas para a análise foram fase móvel: água e acetonitrila na proporção 50:50 (v/v), respectivamente, fluxo: 0,8mL min $^{-1}$; volume de injeção: $20 \mu \mathrm{L}$; temperatura da coluna: $30^{\circ} \mathrm{C}$; comprimento de onda: $254 \mathrm{~nm}$; e tempo de retenção de $7 \mathrm{~min}$. A quantificação foi realizada pela comparação das áreas obtidas nos cromatogramas pelo método de calibração externa.

Os dados foram submetidos à análise de variância. Para a concentração no solo do diuron nas diferentes épocas de coletas foram feitas análises de regressão. Ajustou-se equação exponencial: $\mathrm{Y}=\mathrm{A} * \exp \left(-\mathrm{B}^{*} \mathrm{~T}\right)$, em que $\mathrm{T}$ - representa o tempo, Y - concentração do diuron ainda presente no solo, A - concentração inicial do diuron no solo, e B - constante de dissipação. Para as demais variáveis, as médias foram comparadas utilizando-se o teste de Tukey, adotando-se $5 \%$ de probabilidade para todos os testes.

\section{RESULTADOS E DISCUSSÃO}

O teor do diuron reduziu exponencialmente ao longo do tempo para todos os solos avaliados. A sua dissipação foi mais intensa no $\mathrm{LV} \mathrm{pH} \mathrm{6,2} \mathrm{e} \mathrm{LA} \mathrm{pH}$ 6,3 com a constante de dissipação de -0,0174 e -0,0153, respectivamente. Para o LVA pH 5,0, LVA pH 5,9 e LV pH 5,0 , a taxa de dissipação foi menor, em torno de $-0,008$. A meia-vida estimada do diuron foi menor em solos em que a taxa de dissipação foi mais intensa, 40 dias para LV pH 6,2 e 46 dias para o LA pH 6,3 e, nos demais solos, os valores de meia-vida foram de 89, 90 e 91 para o LV pH 5,0, LVA pH 5,9 e LVA pH 5,0, e respectivamente (Figura 1).

A dissipação mais rápida do diuron no LA pode estar relacionada com a maior atividade microbiana nesse solo, pois, dentre os solos estudados, esse é o que apresenta a maior fertilidade (Tabela 1). De maneira semelhante, a calagem no LV pode ter favorecido maior atividade dos microrganismos, o que reduziu a meia-vida do herbicida nesses solos. CARACCIOLO et al. (2005) verificaram que a meia-vida do diuron em solo esterilizado e não esterilizado foram de 15 e 129 dias, respectivamente, demonstrando assim a importância dos microrganismos na dissipação desse herbicida.

A dissipação do diuron também é favorecida em áreas em que esse herbicida foi aplicado sucessivas vezes. Esse fato foi confirmado por ROUCHAUD et al. (2000) os quais avaliaram sua dissipação em áreas com histórico de aplicação e encontraram a meiavida de 37 dias. Entretanto, quando compararam os resultados com os obtidos de área onde o herbicida foi aplicado pela primeira vez, observaram meia-vida de 81 dias. Os autores atribuíram essa menor meia-vida do diuron a uma adaptação dos microrganismos do solo, favorecendo assim a sua biodegradação. Além desse fator, outros observados em condições de campo, como os climáticos, podem influenciar a persistência do herbicida no solo (DORES et al., 2009). Esses autores encontraram meia-vida do diuron em condições de campo em Latossolo Vermelho de 15 dias. Nessas condições, a menor meia-vida observada pode ser atribuída a outros meios de remoção do herbicida do perfil do solo, como lixiviação e escorrimento superficial. 
Neste trabalho, a presença do diuron foi detectada até 156DAA em todos os solos, por meio da análise cromatográfica (Figura 1), da manifestação de sintoma de intoxicação da espécie bioindicadora ou da percentagem de acúmulo de matéria seca em relação à testemunha (Tabela 2). O LVA é o solo em que as concentrações do herbicida são mais elevadas. Entretanto, o LVA pH 5,0 foi o solo em que os sintomas de intoxicação foram menos severos na última avaliação, e também foi o solo em que as plantas tiveram maior acúmulo de matéria seca, isso devido, provavelmente, ao fato de esse solo apresentar um maior coeficiente de sorção do herbicida ao solo. Nessa situação, apesar de presente no solo, o diuron está retido aos coloides do solo, portanto, sendo pouco absorvido pelas plantas. $\mathrm{O}$ diuron pode apresentar longa persistência no solo. Em experimento a campo, na cultura da cana-deaçúcar, STORK et al. (2008) observaram a presença do herbicida até 267 dias após a aplicação e estimaram uma longa persistência dos metabólitos da degradação desse agrotóxico, principalmente do 3-(3,4-diclorofenil)-3metilureia (DCMPU).

Os solos com os maiores teores de areia, LA pH 6,3 e LV pH 6,2, apresentaram as menores concentrações do diuron aos 156DAA (Figura 1). A dissipação nos solos arenosos é mais intensa, pois o herbicida é menos sorvido do que em solos argilosos, tornando assim mais disponíveis às plantas e aos microrganismos para a degradação da molécula (INOUE et al., 2011).

A intoxicação da espécie bioindicadora pelo diuron foi elevada até aos 96DAA, para todos os solos. No LV, aos 156DAA, foi ainda alta, independentemente do $\mathrm{pH}$ e no LVA e LA os sintomas foram menos intensos (Tabela 2). A avaliação da presença de herbicidas com uso de espécie bioindicadoras é largamente utilizado (INOUE et al., 2008; ANDRADE et al., 2010; MELO et al., 2010), entretanto, sabe-se que a quantificação do herbicida é limitada por essa técnica. Outra limitação é que, em solos onde o herbicida encontra-se sorvido fortemente pelos coloides, esses compostos não ficam passíveis de serem absorvidos pelas plantas. Isso foi possível verificar no LVA, que apresentou menores valores de intoxicação na última avaliação, embora as concentrações do diuron nesse solo, determinadas por cromatografia, tenham sido as mais elevadas entre os solos estudados (Figura 1). O LVA

Tabela 2 - Intoxicação e matéria seca de plantas de pepino, no Latossolo Amarelo (LA), Latossolo Vermelho Amarelo (LVA) e Latossolo Vermelho (LV), semeadas nos diferentes dias após a aplicação do diuron, e avaliadas aos 21 dias após a emergência das plântulas.

\begin{tabular}{|c|c|c|c|c|c|}
\hline Dias após a aplicação & LVA pH 5,0 & LVA pH 5,9 & $\mathrm{LV}$ pH 5,0 & LV pH 6,2 & LA pH 6,3 \\
\hline 1 & $100,0 \mathrm{Aa}$ & $100,0 \mathrm{Aa}$ & $100,0 \mathrm{Aa}$ & $100,0 \mathrm{Aa}$ & $100,0 \mathrm{Aa}$ \\
\hline 8 & $96,7 \mathrm{Aa}$ & $100,0 \mathrm{Aa}$ & $100,0 \mathrm{Aa}$ & $100,0 \mathrm{Aa}$ & $100,0 \mathrm{Aa}$ \\
\hline 15 & $100,0 \mathrm{Aa}$ & $100,0 \mathrm{Aa}$ & $100,0 \mathrm{Aa}$ & $100,0 \mathrm{Aa}$ & $100,0 \mathrm{Aa}$ \\
\hline 22 & $100,0 \mathrm{Aa}$ & $100,0 \mathrm{Aa}$ & $100,0 \mathrm{Aa}$ & $100,0 \mathrm{Aa}$ & $100,0 \mathrm{Aa}$ \\
\hline 36 & $95,0 \mathrm{Aa}$ & $100,0 \mathrm{Aa}$ & $100,0 \mathrm{Aa}$ & $100,0 \mathrm{Aa}$ & $100,0 \mathrm{Aa}$ \\
\hline 66 & $88,3 \mathrm{Bb}$ & $100,0 \mathrm{Aa}$ & $100,0 \mathrm{Aa}$ & $100,0 \mathrm{Aa}$ & $100,0 \mathrm{Aa}$ \\
\hline 96 & $97,6 \mathrm{Aa}$ & $100,0 \mathrm{Aa}$ & $100,0 \mathrm{Aa}$ & $100,0 \mathrm{Aa}$ & $100,0 \mathrm{Aa}$ \\
\hline 156 & $30,0 \mathrm{Cc}$ & $56,7 \mathrm{Bb}$ & $100,0 \mathrm{Aa}$ & $100,0 \mathrm{Aa}$ & $55,0 \mathrm{Bb}$ \\
\hline $\mathrm{CV}(\%)$ & 2,65 & & & & \\
\hline \multicolumn{6}{|l|}{ Dias após a aplicação } \\
\hline 1 & $0 \mathrm{Aa}$ & $0 \mathrm{Aa}$ & $0 \mathrm{Aa}$ & $0 \mathrm{Aa}$ & OAa \\
\hline 8 & $16,3 \mathrm{Bb}$ & $0 \mathrm{Aa}$ & $0 \mathrm{Aa}$ & $0 \mathrm{Aa}$ & $0 \mathrm{Aa}$ \\
\hline 15 & $0 \mathrm{Aa}$ & $0 \mathrm{Aa}$ & $0 \mathrm{Aa}$ & $0 \mathrm{Aa}$ & 0Aa \\
\hline 22 & $0 \mathrm{Aa}$ & $0 \mathrm{Aa}$ & $0 \mathrm{Aa}$ & $0 \mathrm{Aa}$ & 0Aa \\
\hline 36 & $47,5 \mathrm{Bc}$ & 0Aa & 0Aa & 0Aa & 0Aa \\
\hline 66 & $68,8 \mathrm{Bd}$ & $0 \mathrm{Aa}$ & 0Aa & 0Aa & OAa \\
\hline 96 & $37,9 \mathrm{Bc}$ & $0 \mathrm{Aa}$ & $0 \mathrm{Aa}$ & $0 \mathrm{Aa}$ & OAa \\
\hline 156 & $63,2 \mathrm{Dd}$ & $31,8 \mathrm{Bb}$ & $0 \mathrm{Aa}$ & $0 \mathrm{Aa}$ & $42,5 \mathrm{Cb}$ \\
\hline $\mathrm{CV}(\%)$ & 58,63 & & & & \\
\hline
\end{tabular}

Médias seguidas pelas mesmas letras minúsculas nas colunas e maiúsculas nas linhas, dentro de cada variável, não diferem entre si pelo teste de Tukey a 5\% de probabilidade. 


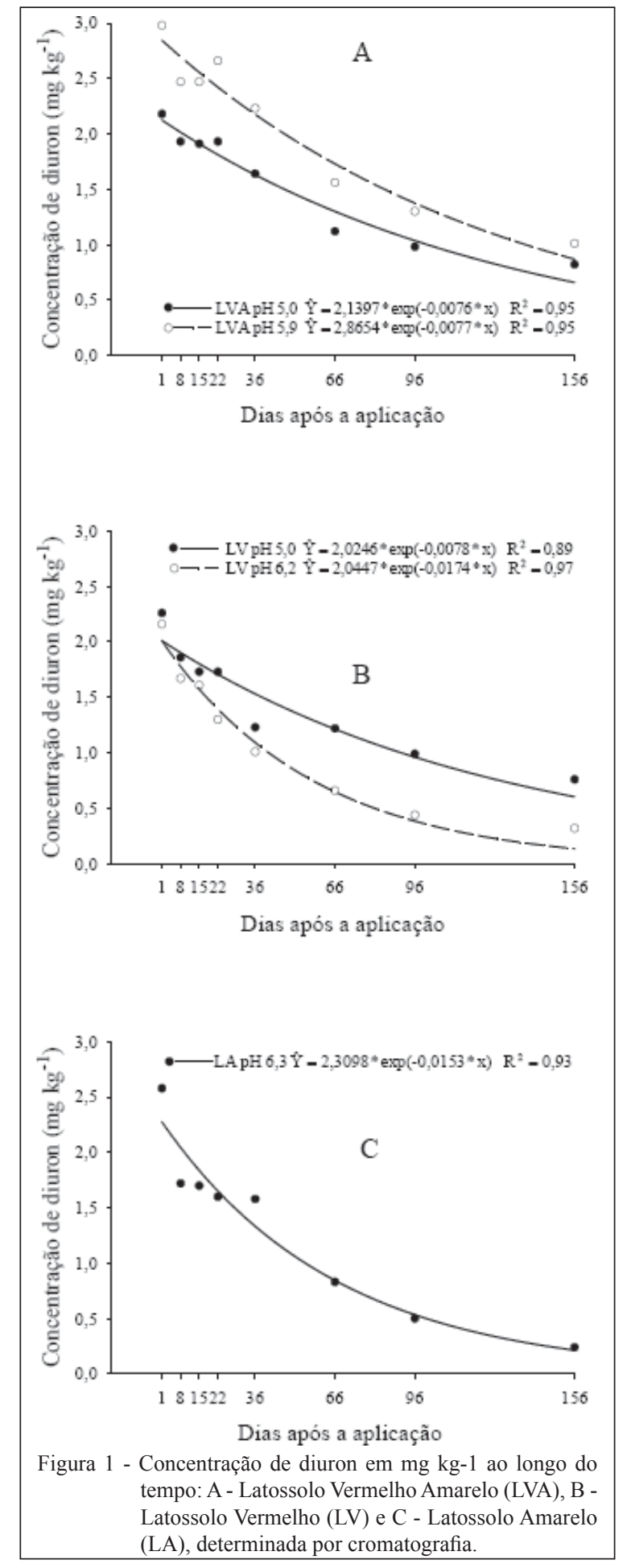

é o solo que apresentou os maiores teores de matéria orgânica (Tabela 1). Com isso, houve maior sorção do herbicida e uma menor concentração do diuron na solução do solo.

A formação de resíduos sorvidos ao solo tem como consequência a diminuição da degradação do agrotóxico e a perda da atividade biológica (CALDERBANK, 1989). A sorção do diuron está diretamente relacionada com os teores de matéria orgânica, principalmente através de ligações hidrofóbicas entre a molécula do herbicida com matéria orgânica (GONZÁLES-PRADAS et al., 1998; LIU et al., 2010).

O uso do diuron em solos como Latossolo Vermelho, que apresentou baixa capacidade de retenção e elevada movimentação do herbicida, associado à elevada persistência desse composto (Figura 1B), pode favorecer a contaminação de águas subterrâneas.

\section{CONCLUSÃO}

A persistência do diuron é dependente dos atributos físicos e químicos dos solos, sendo a sua dissipação menor em solos com maior teor de matéria orgânica. A correção do $\mathrm{pH}$ do solo favorece a sua degradação apenas em solos arenosos com baixo teor de matéria orgânica. A meia-vida diuron nos solos estudados variou de 40 a 91 após sua aplicação e os testes biológicos se mostraram eficientes e complementares às análises cromatográficas neste estudo.

\section{AGRADECIMENTOS}

Ao Conselho Nacional de Desenvolvimento Científico e Tecnológico (CNPq), pelo apoio financeiro.

\section{REFERÊNCIAS}

ANDRADE, S.R.B. et al. Meia-vida do ametryn em argissolo vermelho-amarelo e latossolo vermelho-amarelo, com diferentes valores de pH. Planta Daninha, v.28, n.2, p.375-383, 2010. Disponível em: <http://www.scielo.br/scielo.php?pid=S010083582010000200017\&script=sci_arttext $>$. Acesso em: 08 mar. 2012. doi: 10.1590/S0100-83582010000200017.

CALDERBANK, A. The occurrence and significance of bound pesticide residues in soil. Reviews of Environmental Contamination and Toxicology, v.108, p.71-103, 1989.

CARACCIOLO, A.B. et al. Degradation and leaching of the herbicides metolachlor and diuron: a case study in an area of Northern Italy. Environmental Pollution, n.134, p.525-534, 2005. Disponível em: <http://www.sciencedirect.com/science/ article/pii/S0269749104003562>. Acesso em: 08 mar. 2012. doi: 10.1016/j.envpol.2004.08.014.

COBBUCI, T.; MACHADO, E. Seletividade, eficiência de controle de plantas daninhas e persistência no solo de imazamox aplicado na cultura do feijoeiro. Planta Daninha, v.17, n.3, p.419-432, 1999. Disponível em: <http://www.scielo.br/scielo.php?pid=S0100$83581999000300009 \&$ script $=$ sci_abstract\&tlng $=\mathrm{es}>$. Acesso em: 08 mar. 2012. doi: 10.1590/S0100-83581999000300009. 
DORES, E.F.G.C. et al. Environmental behaviour of metolachlor and diuron in a tropical soil in the central region of Brazil. Water Air Soil Pollut, v.197, n.1-4, p.175-183, 2009. Disponível em: $<$ http://www.springerlink.com/content/r267801051q58801/>. Acesso em: 08 mar. 2012. doi: 10.1007/s11270-008-9801-1.

EUROPEAN WEED RESEARCH COUNCIL (EWRC). Report of the $3^{\text {rd }}$ and $4^{\text {th }}$ meetings of EWRC - Comittee of Methods in Weed Research. Weed Research, v.4, n.1, p.88, 1964.

FERRI, M.V.W.; VIDAL, R.A. Persistência do herbicida acetochlor em função de sistemas de preparo e cobertura com palha. Ciência Rural, v.33, n.3, p.399-404, 2003. Disponível em: $<$ http://www.scielo.br/scielo.php?script=sci arttext\&pi $\mathrm{d}=\mathrm{S} 0103-84782003000300002>$. Acesso em: 08 mar. 2012. doi: $10.1590 / \mathrm{S} 0103-84782003000300002$.

GONZÁLEZ-PRADAS, E. et al. Sorption and leaching of diuron on natural and peat-amended calcareous soil from Spain. Water Research, v.32, n.9, p.2814-2820, 1998. Disponível em: <http:// www.sciencedirect.com/science/article/pii/S0043135498000219>. Acesso em 08 mar. 2012. doi: 10.1016/S0043-1354(98)00021-9.

HAGER, A.; NORDBY, D. Herbicide persistence and how to test for residues in soils. In: BISSONNETTE. S. Illinois agricultural pest management handbook. Chicago: University of Illinois extension, 2004. p.343-350.

INOUE, M.H. et al. Lixiviação e degradação de diuron em dois solos de textura contrastante. Acta Scientiarum Agronomy, v.30, n.5, p.631-638, 2008. Disponível em: <http://periodicos.uem.br/ ojs/index.php/ActaSciAgron/article/view/5963/5963>. Acesso em: 08 mar. 2012. doi: 10.4025/actasciagron.v30i5.5963.

INOUE, M.H. et al. Efeito residual de herbicidas aplicados em pré-emergência em diferentes solos. Planta Daninha, v.29, n.2, p.429-435, 2011. Disponível em: <http://www.scielo. br/scielo.php? pid $=$ S0100-83582011000200021\& script $=$ sci arttext>. Acesso em: 10 dez. 2011. doi: 10.1590/S010083582011000200021 .

KASOZI, G.N. et al. Characterization of adsorption and degradation of diuron in cabonatic and noncarbonatic soils. Journal of Agricultural and Food Chemistry, v.58, n.2, p.1055-1061, 2010 Disponível em: <http://pubs.acs.org/doi/full/10.1021/jf902792p>. Acesso em: 08 mar. 2012. doi: 10.1021/jf902792p.
NAKAGAWA, L.E.; ANDRÉA, M.M. Degradação e formação de resíduos não-extraíveis ou ligados do herbicida atrazina em solo. Pesquisa Agropecuária Brasileira, v.35, n.8, p.1509-1515, 2000. Disponível em: <http://www.scielo.br/scielo.php?pid=S0100204X2000000800002\&script $=$ sci_arttext $>$. Acesso em: 08 mar. 2012. doi: 10.1590/S0100-204X2000000800002.

LIU, Y. et al. Adsorption and desorption behavior of herbicide diuron on various Chinese cultivated soils. Journal of Hazardous Materials, v.178, n.1-3, p.462-468, 2010. Disponível em: <http:// www.sciencedirect.com/science/article/pii/S030438941000141X>. Acesso em: 10 dez. 2011. doi: 10.1016/j.jhazmat.2010.01.105.

MANCUSO, M.A.C. et al. Efeito residual de herbicidas no solo ("Carryover"). Revista Brasileira de Herbicidas, v.10, n.2, p.151-164, 2011. Disponível em: <http://www.rbherbicidas.com. br/index.php/rbh/article/view/106>. Acesso em: 10 dez. 2011.

MELO, C.A.D. et al. Efeito residual de sulfentrazone, isoxaflutole e oxyfluorfen em três solos. Planta Daninha, v.28, n.4, p.835-842, 2010. Disponível em: $<$ http://www.scielo.br/scielo.php?pid=S010083582010000400017\&script=sci_arttext $>$. Acesso em: 08 mar. 2011. doi: 10.1590/S0100-83582010000400017.

RODRIGUES, B.N.; ALMEIDA, F.S. Guia de herbicidas. 6.ed. Londrina: Grafmarke, 2011. 697p.

ROUCHAUD, J. et al. Soil dissipation of diuron, chlorotoluron, simazine, propyzamide, and diflufenican herbicides after repeated applications in fruit tree orchards. Archives of Environmental Contamination and Toxicology, v.39, n.1,p.60-65,2000. Disponível em: <http://www.springerlink.com/content/gku9gpfcl16g96p6/>. Acesso em: 08 mar. 2012. doi: 10.1007/s002440010080.

SINDAG (SINDICATO NACIONAL DA INDÚSTRIA DE PRODUTOS PARA DEFESA AGRÍCOLA). Disponível em: $<$ http://www.sindag.com.br/conexao/anteriores/conexao_n22. pdf $>$. Acesso em: 03 maio, 2011.

STORK, P.R. et al. The environmental fate of diuron under a conventional production regime in a sugarcane farm during the plant cane phase. Pest Management Science, v.64, p.954-963, 2008. Disponível em: $<$ http://dx.doi.org/10.1002/ps.1593>. Acesso em: 08 mar. 2012. doi: 10.1002/ps.1593. 